\title{
BMJ Open Role of cumulative biological risk in mediating socioeconomic disparities in cognitive function in the elderly: a mediation analysis
}

\author{
Jimmy Akrivos (D) , ${ }^{1}$ Carolyn Wei Zhu, ${ }^{2}$ Vahram Haroutunian ${ }^{3}$
}

To cite: Akrivos J, Zhu CW, Haroutunian V. Role of cumulative biological risk in mediating socioeconomic disparities in cognitive function in the elderly: a mediation analysis. BMJ Open 2020;10:e035847. doi:10.1136/ bmjopen-2019-035847

- Prepublication history and additional material for this paper are available online. To view these files, please visit the journal online (http://dx.doi org/10.1136/bmjopen-2019035847).

Received 18 November 2019 Revised 28 July 2020 Accepted 05 August 2020

\section{Check for updates}

\section{Author(s) (or their} employer(s)) 2020. Re-use permitted under CC BY-NC. No commercial re-use. See rights and permissions. Published by BMJ.

1Department of Psychiatry, Alzheimer's Disease Research Center, Icahn School of Medicine at Mount Sinai, New York, New York, USA

${ }^{2}$ Geriatrics and Palliative Medicine, Icahn School of Medicine at Mount Sinai, New York, New York, USA

${ }^{3}$ Psychiatry and Neuroscience, Icahn School of Medicine at Mount Sinai, New York, New York, USA

Correspondence to Dr Jimmy Akrivos; jimmy.akrivos@mssm.edu

\section{ABSTRACT}

Objectives To evaluate whether allostatic load (AL), a measure of cumulative biological risk, fully or partially mediates observed socioeconomic status (SES) differences in cognitive function in the elderly.

Design Cross-sectional mediation analysis.

Setting Community-dwelling US elderly who participated in the National Health and Nutrition Examination Survey (NHANES).

Participants The NHANES uses a complex, multistage, probability sampling design to select a nationally representative sample. Of the 4976 elderly (60 years or older) who were selected, 3234 agreed to participate in the household and medical exam interviews $(65 \%$ response rate).

\section{Primary and secondary outcome}

measures Performance on the Digit Symbol Substitution Test (DSST) - a measure of cognitive function.

Results Relative to participants with the lowest level of education or family income, participants who were college graduates $(\beta=24.4,95 \% \mathrm{Cl} 22$ to $26.8, p<0.0001)$ or in the highest income quartile $(\beta=17.3,95 \% \mathrm{Cl} 15.2$ to 19.4 , $\mathrm{p}<0.0001$ ) had the highest DSST scores and the least AL burden $(\beta=-0.72,95 \% \mathrm{Cl}-0.98$ to -0.47 and $\beta=-0.82$, $95 \% \mathrm{Cl}-1$ to $-0.57 ; p<0.0001$, respectively). Although,

$A L$ was significantly negatively associated with cognitive performance ( $\beta=-1,95 \% \mathrm{Cl}-1.4$ to $-0.5, p<0.0001)$, it mediated at most $4.5 \%$ of the SES effect on DSST performance.

Conclusions The findings suggest that $\mathrm{AL}$, as measured by a summary index of parameters for cardiovascular function, metabolism and chronic inflammation, is not a significant mediator of SES-related differences in cognitive function in the elderly. Further efforts are required to elucidate the exact physiological pathways and mechanisms through which SES impacts cognitive function in late life.

\section{INTRODUCTION}

The number of people afflicted with dementia is projected to almost triple by 2050 primarily due to large increases in the elderly population and particularly the oldest old. ${ }^{12}$ As a result, the public health, social and economic burden of caring for those with late-life cognitive disorders will continue to grow. ${ }^{3-5}$ After
Strengths and limitations of this study

- Methodological advantages over previous studies consist of the use of a large, nationally representative sample of US elderly and multiple imputations to account for missing data.

- The analyses used a comprehensive Allostatic Load (AL) Index, simultaneous measurements of socioeconomic status and cognitive function, and controlled for important demographic confounders.

- Because this was a cross-sectional analysis, we cannot rule out whether changes in the causal relations in the mediation model over time may have led to an underestimation of the mediation effect

- We also cannot rule out potential confounding by genes, such as apolipoprotein E (APOE), or psychosocial factors, such as depression, that influence both AL biomarkers and late-life cognitive outcomes.

more than 30 years of efforts, effective interventions remain elusive and there is growing recognition that brain changes related to the most common cause of dementia may start decades before clinical symptoms emerge. ${ }^{6-8}$ Consequently, efforts to identify modifiable risk factors that may thwart or delay the onset of late-life cognitive impairment continue to attract considerable scientific interest. Several recent epidemiological studies have reported that the age-adjusted risk of developing dementia in developed countries may be in decline, which has coincided with improvements in education and the reduced prevalence of vascular risk factors at the population level. ${ }^{9-11}$ The decline of dementia prevalence and incidence appears to be concentrated in those with higher levels of education, or without a history of stroke. This evidence suggests that better management of a range of cardiovascular risk factors and lifelong learning and its associated socioeconomic benefits may be modifiable pathways affecting risk of cognitive impairment in late life. ${ }^{12}$ 
Socioeconomic status (SES) as measured by income, education or occupation has been widely associated with cognitive health outcomes in the elderly. ${ }^{13}{ }^{14}$ Several systematic reviews and meta-analyses have found that lower educational attainment is a risk factor for cognitive decline and dementia. ${ }^{15} 16$ A recent review of potentially modifiable risk factors for Alzheimer's disease (AD) estimated that, out of all the risk factors considered, a reduction in the prevalence of low education would make the largest contribution to a reduction of $\mathrm{AD}$ cases worldwide. ${ }^{17}$ The main pathways, which have been studied with respect to SES differences in cognitive health in the elderly include differential exposure to greater and higher quality mental and physical stimulation both early and throughout the life course, material conditions (eg, nutrition and living conditions), psychosocial factors (eg, psychological or social stress) and health behaviours (eg, smoking and lack of exercise). ${ }^{18-20}$ Biological pathways have been studied mainly in the context of individual risk parameters and clinically manifest disease. However, less is known about the role of cumulative biologic burden across multiple systems-including subclinical dysregulation-in mediating SES differences in health outcomes in the elderly and particularly cognitive outcomes.

Impaired glucose metabolism, midlife obesity and hypertension, dyslipidaemia and chronic inflammation have all been linked with risk of dementia, cerebrovascular pathological changes associated with vascular dementia and the hallmark neuropathological features associated with $\mathrm{AD} .{ }^{21-24} \mathrm{~A}$ significant feature of the biological dysregulation associated with these risk factors is that it not only predicts cognitive and overall health risks, but it also varies by SES, with lower SES groups exhibiting the highest levels of dysregulation. ${ }^{25} \mathrm{~A}$ higher prevalence of these risk factors has also been found as early as early childhood in lower SES groups and this disparity persists throughout life. ${ }^{26}$ This raises the prospect of a long-term process of cumulative biological 'wear and tear' across multiple physiological systems that may help explain SES disparities in cognitive function in late life.

'Allostatic load' (AL), proposed by McEwen, Stellar, Wingfield and others, is a multisystems view of the cumulative physiological toll that may be exacted on the body over the life course. In response to environmental stressors and the need for adaptation to those stressors, the brain determines appropriate behavioural and physiological responses. These responses occur through the neuroendocrine, autonomic, immune, and metabolic systems and their mediators, which in turn either directly or through epigenetic programmes alter cellular and organ function. Although this process may be adaptive initially, chronic exposure to limited resources, toxic or unhealthy living conditions, negative life events and the chronic activation of physiological responses to these stressors eventually damage the body's ability to turn stress mediators on or off as needed and lead to dysregulation and lasting pathophysiological changes in cardiovascular, metabolic and immune system function. ${ }^{27}$ The differential accumulation of this biological dysregulation correlates with differences in exposure to adverse circumstances and is posited to mediate SES-related inequalities in physical and cognitive health. This process may begin in childhood and combine with adult and genetic risk factors to exacerbate risk of age-related disease over the life course. For example, research suggests that exposure to childhood socioeconomic disadvantage, maltreatment or social isolation is associated with elevated risk for depression, inflammation and cardiovascular risk factors in adulthood, all of whom have been associated with risk of cognitive decline in late life. ${ }^{28}$ These effects appear to be additive to the effects of risk factors and disease mechanisms that emerge in adulthood in the form of poor cardiometabolic health, which may then affect cognition. More likely, AL has a cumulative impact throughout the life course, directly affecting brain development in early life, as well as, indirectly by resulting in poorer overall health across the life span. ${ }^{29} 30$

The earliest efforts to operationalise AL were reported by the MacArthur Studies of Successful Aging and included primary mediators and secondary markers of physiological health across a range of regulatory systems. ${ }^{31}$ Primary mediators were circulating hormones that play a role in the body's stress response through the hypothalamic-pituitary-adrenal axis and sympathetic nervous system. These mediators interact with each other, body organs and tissue substrates to influence peripheral biological function, which under chronic stress conditions may lead to lasting pathophysiological processes affecting cardiovascular, metabolic, immune and central nervous system function. ${ }^{27} 32$ The main differences between AL and the more traditional focus on individual parameters of biological risk are that it considers overall physiological dysregulation and that it takes into account even relatively modest forms of dysregulation that do not necessarily meet clinically significant thresholds or clinical criteria for disease classification. There exists empirical evidence that such an index of cumulative biological dysregulation can help explain SES-related differences in physical function and mortality better than individual risk parameters. ${ }^{33} 34$ However, to date no studies have assessed the mediating role of cumulative biologic risk in SES disparities in cognitive health in the elderly.

The goal of this study is to evaluate whether AL fully or partially mediates observed SES differences in cognitive function in a nationally representative sample of US elderly. We hypothesised that: (1) without controlling for AL, low SES will be associated with poorer cognitive function; (2) low SES will be associated with higher AL; (3) high AL will be associated with poorer cognitive function, controlling for SES and it will partially mediate the relationship between SES and cognitive function and (4) the mediation effect will be statistically and clinically significant. 


\section{METHODS}

\section{Study sample}

The current research is a secondary data analysis, which combined available data from a cross-sectional, nationally representative sample of elderly (60 years or older) from the National Health and Nutrition Examination Survey (NHANES) 1999-2000 and 2001-2002 cycles. ${ }^{35}$ The 1999-2002 period is the most recent for which cognitive testing data on the elderly NHANES subsample were available at the start of this analysis. The NHANES programme began in 1959 as a series of periodic health surveys that combine in-home interviews on participant demographics, socioeconomic, dietary, self-reported health, physical and cognitive functioning, household conditions and family information followed by standardised physical examinations and laboratory tests. Since 1999, it has been conducted annually (data are released in 2-year cycles) using a stratified, four stage, probability sampling design to select a nationally representative sample of US households. ${ }^{36}$ Approximately 30 counties are visited during each 2-year survey cycle (15 on an annual basis) and participants are recruited from all regions of the USA. ${ }^{35}$ In the 1999-2002 period, certain subgroups of particular public health interest were oversampled, including low-income persons (beginning in 2000), and persons aged 70 and over, among others. ${ }^{36}$ Informed consent is obtained during the home interview and all data that are collected and made publically available are deidentified by the National Center for Health Statistics (NCHS) prior to its release. ${ }^{37}$ For additional information on the NHANES Study design, sampling procedures, study protocols and data collection please see the online supplemental materials: Methods.

\section{Cognitive Function Questionnaire}

Cognitive function in this sample of elderly was assessed using the Digit Symbol Substitution Test (DSST)-a component of the Wechsler Adult Intelligence Test (WAIS) ${ }^{38}$ As a subtest of the WAIS, the DSST has undergone repeated and rigorous psychometric validation such as test-retest reliability and discriminant validity in a range of patient samples. ${ }^{39}$ It has been found to be a sensitive measure of age, cardiovascular, cerebrovascular and dementia-related changes in motor speed, attention and visuospatial functions with test-retest reliability correlation coefficients in the $0.82-0.88$ range in these clinical and normal populations. ${ }^{38}{ }^{39}$ As such, it has become one of the most commonly used instruments in standardised clinical evaluations in studies of cognitive ageing and dementia. ${ }^{40}$ The test consists of a paper-andpencil task that requires copying as many novel symbols corresponding to numbers as possible in $120 \mathrm{~s}$. Using the key provided at the top of the form, participants draw the symbol under the corresponding number. One point is given for each correctly drawn symbol, with a minimum score of 0 and a maximum-for the version used in the NHANES Survey—of 133, with higher scores indicating better performance. Sample items were provided for

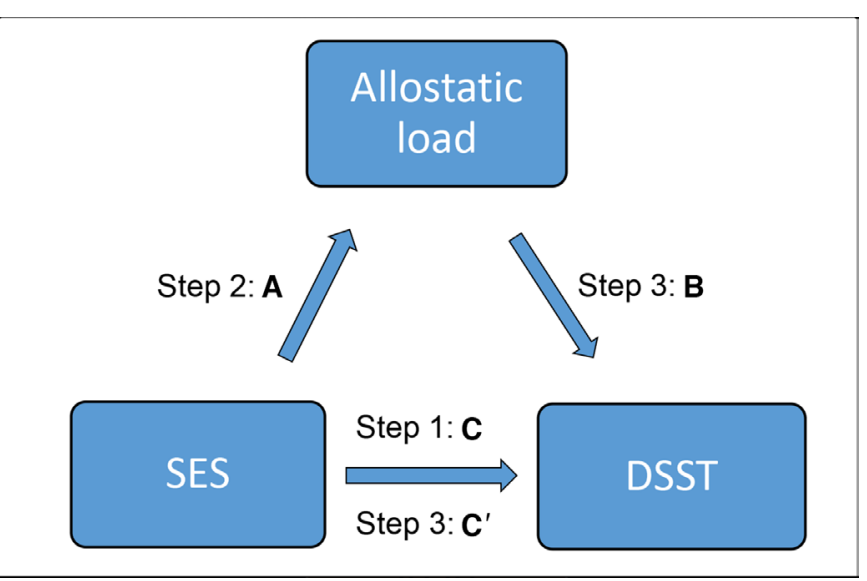

Figure 1 Letters A, B, C and C' represent estimated path parameters. Controlling for participants' age, sex and race/ ethnicity: in step 1, DSST was regressed on SES (C); in step 2, AL was regressed on SES (A) and in step 3, DSST was regressed on SES $\left(C^{\prime}\right)$ and $A L(B)$ simultaneously. $A L$, allostatic load; DSST, Digit Symbol Substitution Test; SES, socioeconomic status.

practice and participants who were unable to complete any of the practice items did not continue with the remainder of the exercise. For a copy of the DSST instrument, see figure 1 in the online supplemental materials.

\section{Socioeconomic status}

The present study focused on SES as defined by selfreported years of education completed and, separately, as the poverty income ratio (PIR), which reflects reported household income relative to the federally defined poverty level for the participant's area of residence and household size. In the NHANES, education is categorised into less than 9th grade, 9th-11th grade, high school (HS) graduate or general equivalency diploma, some college, and college graduate. Because NHANES uses top coding to protect the anonymity of the highest income participants (PIR of 5 or greater), we categorised PIR into four categories based on the quartile cut offs of its empirical distribution, which consisted of PIR values of $<1.3$, $1.3-2.39,2.4-4.18$ and $>4.18$, respectively.

\section{Allostatic load}

The set of parameters used consisted of systolic blood pressure and diastolic blood pressure (DBP) (reflecting cardiovascular health), body mass index and the ratio of waist-to-thigh (WTR) circumference (reflecting longterm metabolism and adipose tissue deposition), highdensity lipoprotein (HDL), total cholesterol (TC) and the ratio of HDL to TC (reflecting lipid metabolism), glycated haemoglobin (HbAlc) (reflecting glucose metabolism), and $\mathrm{C}$ reactive protein (CRP) (as a marker of systemic inflammation). The body measurement and biomarker assay protocols that were used are available on the NHANES website (source: https://www.cdc.gov/ nchs/nhanes/index.htm). ${ }^{37}$

Using the empirical distribution of each parameter, an AL Index for each participant was calculated by adding 
Table 1 Quartile and clinically defined 'high-risk' criteria for allostatic load

\begin{tabular}{|c|c|c|}
\hline Component & $\begin{array}{l}\text { Quartile cut- } \\
\text { offs }^{*}\end{array}$ & $\begin{array}{l}\text { Clinical } \\
\text { cut-offs }\end{array}$ \\
\hline $\begin{array}{l}\text { Systolic blood pressure (mm } \\
\mathrm{Hg})\end{array}$ & 152 & $150^{49}$ \\
\hline $\begin{array}{l}\text { Diastolic blood pressure }(\mathrm{mm} \\
\mathrm{Hg})\end{array}$ & 78 & $90^{49}$ \\
\hline $\mathrm{TC}(\mathrm{mg} / \mathrm{dL})$ & 235 & $240^{50}$ \\
\hline $\mathrm{HDL}(\mathrm{mg} / \mathrm{dL})$ & $<41$ & $<40^{50}$ \\
\hline TC/HDL ratio & 5 & $\begin{array}{l}>5 \text { men, } \\
>4.5 \\
\text { women }^{51}\end{array}$ \\
\hline BMI $\left(\mathrm{kg} / \mathrm{m}^{2}\right)^{\star}$ & $31.3-31.4$ & $\begin{array}{l}30 \\
\text { obesity }^{52}\end{array}$ \\
\hline Waist to thigh ratio & 2.1 & $\mathrm{n} / \mathrm{a}$ \\
\hline Waist (cm) & $\mathrm{n} / \mathrm{a}$ & $\begin{array}{l}>102 \\
\text { men, }>88 \\
\text { women }^{53}\end{array}$ \\
\hline Glycated haemoglobin (\%) & 5.8 & $6.5^{54}$ \\
\hline $\mathrm{CRP}(\mathrm{mg} / \mathrm{dL})$ & $0.64-0.65$ & $0.3^{55}$ \\
\hline
\end{tabular}

*Identical across all imputed datasets, except for BMI and CRP for which the range is provided.

$\mathrm{BMI}$, body mass index; CRP, C reactive protein; HDL, high-density lipoprotein; TC, total cholesterol; TC/HDL, the ratio of TC to HDL.

the number of parameters for which the participant's value fell in the 'highest risk' quartile (ie, above the 75th percentile for all parameters except HDL cholesterol for which below the 25th percentile corresponded to the highest risk). ${ }^{31}$ It was postulated that membership in the high-risk quartile would differentiate those exposed to greater dysregulation relative to the rest of the sample. AL scores could range from 0 (ie, none of the participant's parameters fell in the high-risk quartile) to 9 (ie, all parameters fell in the high-risk quartile). In post hoc analyses, we examined alternative 'high-risk' cut-off values derived from commonly accepted clinical practice guidelines (see 'Post hoc analyses' section for details). Quartilebased and clinically derived 'high-risk' cut-off values are listed in table 1.

\section{Covariates}

All mediation analysis models controlled for participants' age, gender and race/ethnicity. Because NHANES uses top coding to protect the anonymity of participants who are 85 or older, age was categorised into $60-69,70-79$ and 80 or older. Moreover, due to low cell counts, all 68 observations in the other/multiracial category of the race/ethnicity variable were excluded from the analysis, leaving 3166 participants in the dataset.

\section{Statistical analysis}

Of the 4976 elderly who were selected for NHANES 1999-2002, 3706 agreed to participate in the household interview (74.5\% response rate) and 3234 participated in the medical examination (65\% response rate). NHANES participants are assigned individual weights that account for factors that are correlated with propensity for nonresponse to the household and medical examinations (ie, unit non-response), such as age, race, gender, household size, education, self-reported health status and activity level. ${ }^{36}$ Of the 3234 elderly who participated in the household and medical exam interviews, $1280(39.5 \%)$ had missing data on at least one of the variables that were to be used in the mediation analysis (ie, item non-response). Participants with missing data were significantly different with respect to age, race/ethnicity, SES, AL components and cognitive function. As such, 10 multiple imputations using fully conditional specification were used to address potential biases arising from item non-response. ${ }^{41}$

Once the imputation step was completed, the mediation analysis was performed separately in each of the 10 imputed datasets using the SURVEYREG procedure in SAS V.9.4, which takes into account the effect of stratification, cluster sampling and unequal survey weights on the estimation of regression coefficients and variance parameters. The mediation analysis consisted of the multistep procedure of multiple regressions outlined by Baron and Kenny, ${ }^{42}$ which was used to assess whether the mediation model was correctly specified and to estimate the mediating effect of AL. Figure 1 illustrates the recursive structural equation model that was used in steps 1-3 of the mediation analysis. In the first step, performance on the DSST was regressed on SES, controlling for the covariates, but not the mediator (path C). In the second step, AL was regressed on SES, controlling for the covariates (path A). In the third step, cognitive performance on the DSST was regressed on SES (path $\mathrm{C}^{\prime}$ ) and AL (path B) simultaneously, controlling for the covariates. The indirect effect, which is a measure of the mediation effect, was estimated by taking the product of the coefficients for paths a and $\mathrm{b}$ and the Sobel Test was used to determine its statistical significance. $^{43}$ The estimated regression coefficients and associated standard errors obtained from fitting the linear models in steps 1-3 were synthesised into single sets of statistics using the MIANALYZE procedure in SAS V.9.4. Taken together the SURVEYREG and MIANALYZE procedures produced variance estimates that were fully corrected for the complex sample design of the NHANES Survey, as well as, the additional variability introduced by the multiple imputations.

\section{Post hoc analyses}

A series of post hoc analyses considered the role of individual AL components, separately, as mediators of SES effects on cognitive performance and examined a number of alternative AL indices. Specifically, an alternative scoring algorithm considered subjects' self-reported medication use for diabetes, hypertension or high cholesterol, by assigning a point to their AL Score for each medication used, even if these participants' biomarker values for HbA1c, blood pressure or TC did not fall into the 'high-risk' quartile range. ${ }^{44}$ Moreover, consistent 
with previous scoring methods, we also examined alternative 'high-risk' cut-off values derived from commonly accepted clinical practice guidelines. Although the clinically defined "high-risk" criteria were very similar to the quartile-based cut-offs for most parameters, there were notable exceptions for DBP, HbA1c and CRP. Moreover, because evidence-based clinical guidelines for defining high-risk thresholds for WTR do not exist, WHO waist circumference guidelines for men and women were used as an alternative.

\section{Patient and public involvement}

Although, the public was not directly involved in the design of this study, the development of the research question was informed by the needs of patients, caregivers and society to identify potentially modifiable pathways affecting the risk of cognitive impairment in late life. Identifying factors that help explain SES inequalities in the distribution of reduced cognitive function in old age allow government agencies and private sector organisations to establish policies and plan research, education and health promotion programmes that can directly benefit elderly participants in the USA and worldwide.

\section{RESULTS}

Table 2 displays descriptive statistics for the non-multiply imputed sample and the univariate regression of DSST on all analytic variables. Participants who were older, male, non-white, had lower level of education or income, and higher AL had significantly lower DSST scores.

\section{SES and cognitive function}

To test whether SES has an effect on cognitive function that may be mediated by AL (ie, total effect), performance on the DSST was regressed on SES, controlling for the covariates. As expected, level of education and PIR was significantly positively associated with performance on the DSST. As shown in figure 2A,B, relative to participants with the lowest level of education or family income, participants who were college graduates or in the highest PIR quartile had the highest DSST scores $(\beta=24.4,95 \%$ CI 22 to 26.8 and $\beta=17.3,95 \%$ CI 15.2 to $19.4 ; \mathrm{p}<0.0001$, respectively), followed by participants with some college or who were in the third PIR quartile $(\beta=18.6,95 \%$ CI 16.4 to 21 and $\beta=12.5$, 95\% CI 10.2 to 14.8 ; $\mathrm{p}<0.0001$, respectively) and participants who were HS graduates or in the second PIR quartile ( $\beta=15.5,95 \%$ CI 13 to 18 and $\beta=6,95 \%$ CI 3.5 to $8.5 ; p<0.0001$, respectively). Moreover, the DSST Score of participants who completed 9th-11th grade was also significantly higher than that of participants with the lowest level of education $(\beta=8.9,95 \% \mathrm{CI}$ 6.6 to $11.3, \mathrm{p}<0.0001)$. Table 1 , Hypothesis 1 model, in the online supplemental materials lists the synthesised parameter estimates (over all imputations) for the regression of DSST on SES.

\section{SES and allostatic load}

The first hypothesised pathway of the indirect effect of SES on cognitive function postulates that AL changes
Table 2 Descriptive statistics and univariate regression of DSST on analytic variables

\begin{tabular}{|c|c|c|}
\hline Variables & $\%$ or mean (SE) & $\beta$ (SE) \\
\hline \multicolumn{3}{|l|}{ Age } \\
\hline Age 60-69* & 46.3 & $17(0.9)$ \\
\hline Age $70-79^{*}$ & 35.6 & $8.6(1.2)$ \\
\hline Age 80+ & 18.1 & - \\
\hline \multicolumn{3}{|l|}{ Sex } \\
\hline Female† & 56.6 & $1.9(0.8)$ \\
\hline Male & 43.4 & - \\
\hline \multicolumn{3}{|l|}{ Race/Eethnicity } \\
\hline Black* & 8.5 & $-16(1.1)$ \\
\hline Hispanic* & 8.5 & $-15.6(1.4)$ \\
\hline White & 83 & - \\
\hline \multicolumn{3}{|l|}{ Education (nmiss=13) } \\
\hline$<9$ th grade & 15 & - \\
\hline 9th-11th grade* & 17 & $12.6(1.3)$ \\
\hline $\begin{array}{l}\text { High school/general } \\
\text { equivalency diploma* }\end{array}$ & 29 & $20.5(1.3)$ \\
\hline Some college* & 21.5 & $23.2(1.1)$ \\
\hline College graduate* & 17.5 & $29.7(1.4)$ \\
\hline \multicolumn{3}{|l|}{ PIR (nmiss=416) } \\
\hline $\mathrm{PIR}<1.3$ & 24.2 & - \\
\hline PIR 1.3-2.39* & 25.4 & $7.2(1.5)$ \\
\hline PIR 2.4-4.18* & 25.1 & $15.7(1.3)$ \\
\hline $\mathrm{PIR}>4.18^{\star}$ & 25.3 & $21.2(1.2)$ \\
\hline DSST $($ nmiss $=511)$ & $46.3(0.6)$ & - \\
\hline $\begin{array}{l}\text { Allostatic load }{ }^{*} \\
\text { (nmiss }=630)\end{array}$ & $2.4(0.0)$ & $-1.7(0.3)$ \\
\hline
\end{tabular}

${ }^{*} p<0.0001$.

$\mathrm{tp}<0.05$.

DSST, Digit Symbol Substitution Test; nmiss, number missing; PIR, poverty-to-income ratio.

with SES (ie, path a). As hypothesised, SES, as measured either by participants' education or PIR, was significantly negatively associated with AL. Figure 3A,B shows that relative to participants with the lowest level of education or family income, participants who were college graduates or in the highest PIR quartile had the least AL burden $(\beta=-0.72,95 \%$ CI -0.98 to -0.47 and $\beta=-0.82,95 \%$ CI -1 to $-0.57 ; \mathrm{p}<0.0001$, respectively), followed by participants with some college or who were in the third PIR quartile ( $\beta=-0.37,95 \%$ CI -0.6 to $-0.13, p<0.01$; and $\beta=-0.37$, $95 \% \mathrm{CI}-0.67$ to $-0.06, \mathrm{p}<0.05)$ and participants who were HS graduates or in the second PIR quartile $(\beta=-0.19$, $95 \% \mathrm{CI}-0.44$ to $0.04, p=0.11$; and $\beta=-0.17,95 \% \mathrm{CI}-0.42$ to $0.07, \mathrm{p}=0.17)$. The AL burden of participants who completed 9 th-11th grade was virtually the same as that of participants with the lowest level of education $(\beta=0.03$, $95 \%$ CI -0.19 to $0.25, \mathrm{p}=0.79$ ). Table 1 , Hypothesis 2 


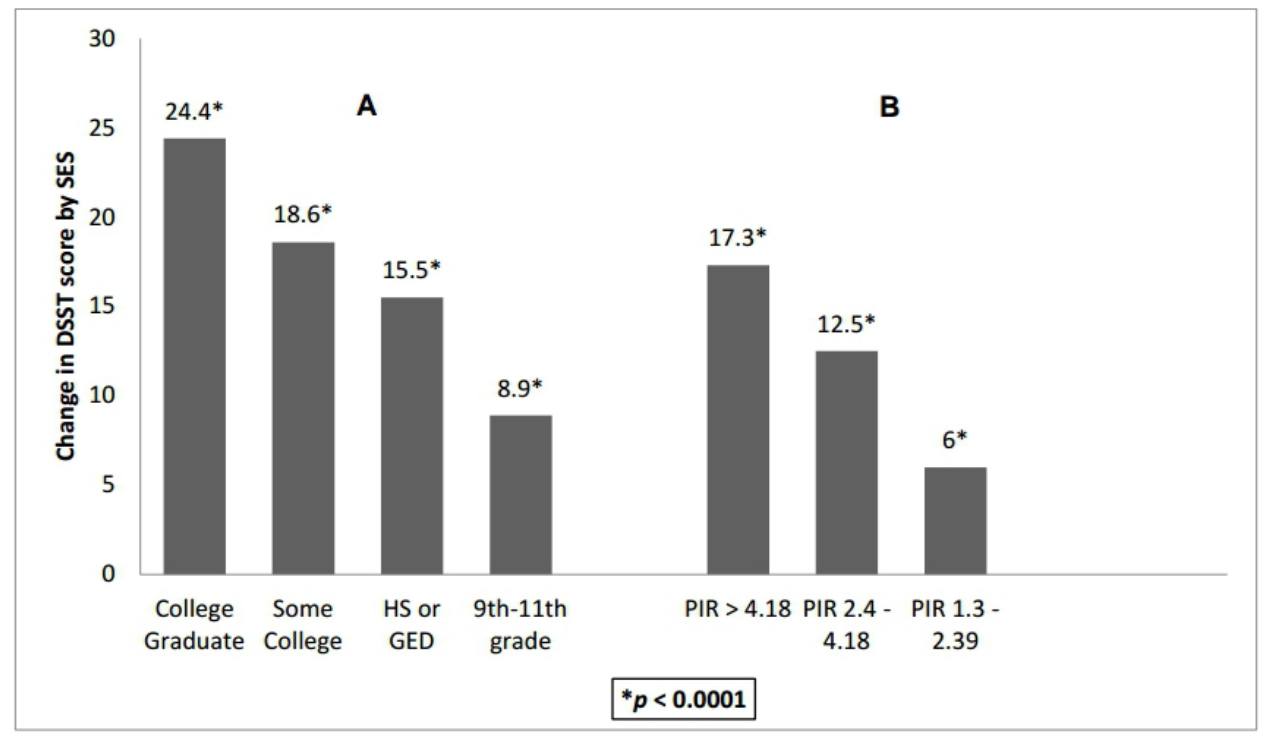

Figure 2 Age, gender and race/ethnicity adjusted change in DSST by SES. DSST, Digit Symbol Substitution Test; GED, general equivalency diploma; HS, high school; PIR, poverty-to-income ratio; SES, socioeconomic status.

model, in the online supplemental materials lists the synthesised parameter estimates (over all imputations) for the regression of AL on SES.

\section{Allostatic load and cognitive function}

To test the second hypothesised pathway through which the indirect effect of SES is postulated to affect cognitive function (ie, path b), performance on the DSST was regressed on SES and AL simultaneously, controlling for the covariates. As postulated, AL was significantly negatively associated with cognitive performance (see figure 4). The magnitude of the association was similar when SES was measured either by participants' education $(\beta=-0.99,95 \% \mathrm{CI}-1.4$ to -0.5$)$ or PIR $(\beta=-0.96,95 \% \mathrm{CI}$ -1.3 to -0.5$)$ at $\mathrm{p}<0.0001$, respectively. Table 1 , Hypothesis 3 model, in the online supplemental materials lists the synthesised parameter estimates (over all imputations) for the regression of DSST on SES, controlling for AL.

\section{Allostatic load as mediator of SES differences in DSST performance}

Controlling for AL attenuated the effect of SES on cognitive performance, although marginally. As shown in figure $5 \mathrm{~A}, \mathrm{~B}$, the parameter estimates for college graduates and those in the highest PIR quartile were reduced by 0.72 and 0.79 DSST points (or by $2.9 \%$ and $4.5 \%$ ), respectively. Parameter estimates for participants with some college and those in the third PIR quartile were reduced by 0.36 and 0.35 DSST points (or by $1.9 \%$ and $2.8 \%$ ), respectively; and for HS graduates and those in the second PIR quartile, parameter estimates were reduced by 0.19 and 0.16 points (or by $1.2 \%$ and $2.7 \%$ ), respectively. The regression coefficient for participants who completed $9^{\text {th }}-11^{\text {th }}$ grade was virtually unchanged after controlling for AL. Although the absolute change in the magnitude of the effect of SES on cognitive performance

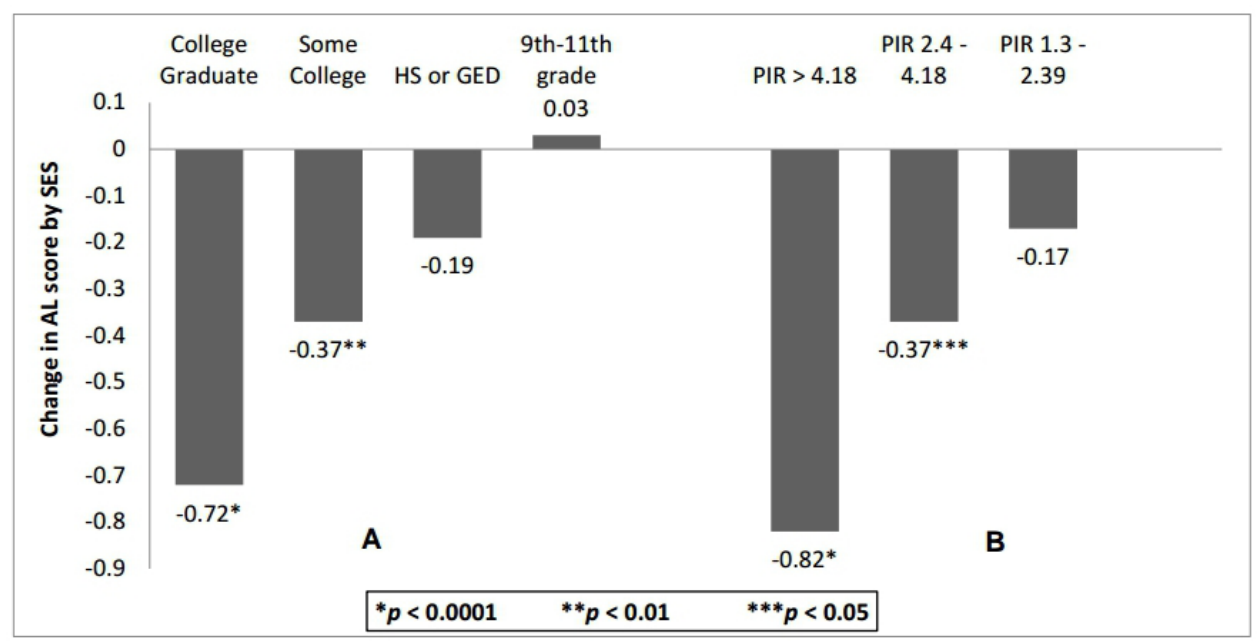

Figure 3 Age, gender and race/ethnicity adjusted change in AL by SES. AL, allostatic load; GED, general equivalency diploma; HS, high school; PIR, poverty-to-income ratio; SES, socioeconomic status. 


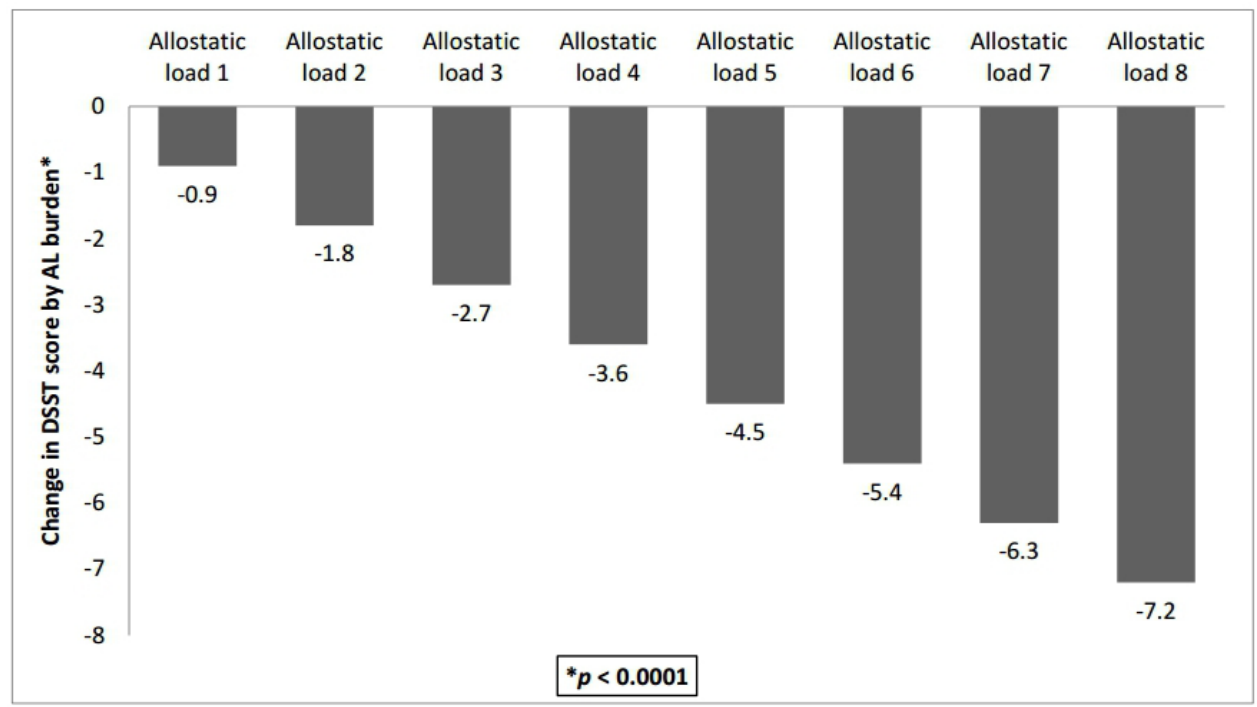

Figure 4 Age, gender and race/ethnicity adjusted change in DSST Score by AL burden. AL, allostatic load; DSST, Digit Symbol Substitution Test.

after controlling for AL was relatively small, Sobel Tests of the indirect effects were statistically significant for college graduates $(\mathrm{ab}=0.72,95 \%$ CI 0.31 to $1.13, \mathrm{p}=0.0006)$ and participants with some college ( $\mathrm{ab}=0.36,95 \% \mathrm{CI} 0.09$ to $0.63, \mathrm{p}=0.011$ ) and for participants in the top two PIR quartiles $(\mathrm{ab}=0.79,95 \% \mathrm{CI} 0.38$ to $1.2, \mathrm{p}=0.0002$ and $\mathrm{ab}=0.35,95 \%$ CI 0.04 to $0.66, \mathrm{p}=0.033$; respectively). All other Sobel Tests were not statistically significant.

\section{Post hoc analyses}

Although, changes in the magnitude of the observed effect of SES on DSST performance after controlling for most individual AL components were marginal, the Sobel Test was statistically significant for WTR and HbA1c. WTR mediated between $2.6 \%$ and $3.2 \%$ of the effect for participants with HS or greater education and $4.0 \%$ of the effect for those in the highest PIR quartile. HbA1c mediated approximately $2 \%$ of the effect in college educated participants and $3.0 \%$ of the effect for participants in the top PIR quartile. Because WTR alone appeared to mediate a similar or greater proportion of SES effects on DSST performance than AL, we examined an alternative AL Index that did not include WTR to ascertain whether

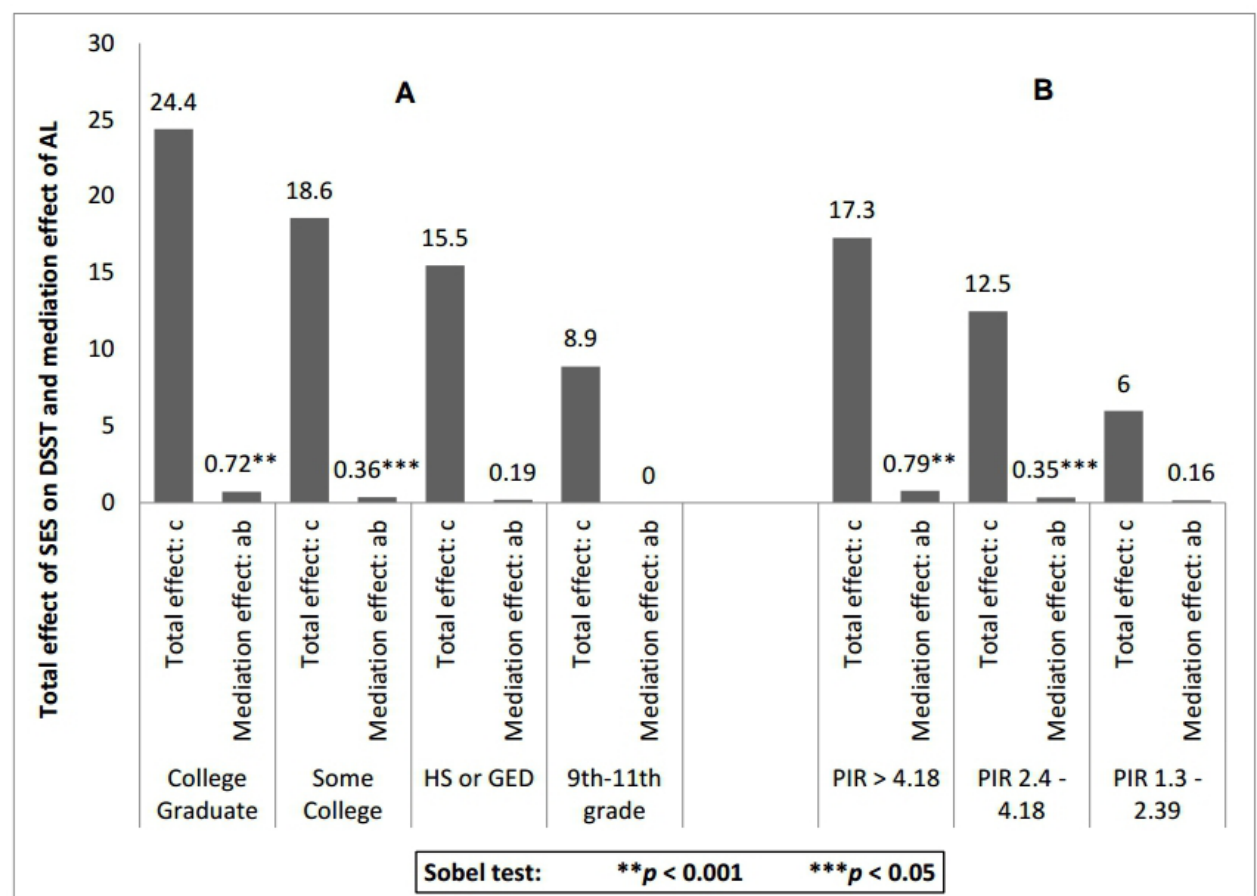

Figure 5 Age, gender and race/ethnicity adjusted change in DSST by SES and mediation effect of AL. AL, allostatic load; DSST, Digit Symbol Substitution Test; GED, general equivalency diploma; HS, high school; PIR, poverty-to-income ratio; SES, socioeconomic status. 
the earlier statistically significant AL findings were due to the effect of WTR. We also examined a second alternative AL Index, which did not include HbA1c. After excluding WTR, or HbAlc, the proportion of the total effect of SES on DSST performance, which was mediated by AL, was now reduced. However, both alternative AL Indices were still found to mediate over $2 \%$ of the effect for college educated participants and between $2.3 \%$ and $3.5 \%$ of the effect for participants in the higher PIR quartiles. In sum, the largest percent mediation of PIR effects was explained by the most comprehensive AL Index, while the largest percent mediation for education effects was explained by WTR alone.

The addition of points for self-reported current medication use to the AL Score led to a small increase (i.e. $<0.7 \%$ ) in the proportion of the total effect for college educated participants and for all PIR quartiles, which was mediated by the index. On the other hand, the use of clinically derived cut-offs led to a marginal decrease in the proportion of the total effect for college educated participants $(\sim 0.5 \%)$ and for all PIR quartiles $(\sim 1 \%)$, which was mediated by the index. In sum, none of the alternative indices altered the results substantially.

In a final set of post hoc analyses, we used data from the NHANES Medical Conditions Questionnaire to examine whether self-reported 'doctor-diagnosed' morbidity modified our findings. We split our sample into a group of 'healthy' participants who had no self-reported doctordiagnosed conditions $(\mathrm{n}=659)$ and a 'morbidity' group who reported at least one medical condition $(n=2507)$ and repeated the Hypothesis 3 analysis separately, within each group. Although in the 'healthy' group, the parameter estimates for the effect of AL on DSST (path b) and for the total and indirect effects of PIR were reduced, in the 'morbidity' group all findings were very similar to those of our main analysis, which did not take medical history into account. See online supplemental table 2 and Post hoc analyses of participants with a medical history section for details.

\section{DISCUSSION}

Overall, changes in DSST Score were strongly positively associated with level of SES and occurred in a nearly linear and dose-dependent fashion. Most notably, even after controlling for participants' age, gender and race/ ethnicity, the DSST Score of college graduates was on the average more than 24 points higher than the score of participants with less than ninth grade education. Similarly, relative to participants with the lowest level of education, the DSST Score was more than 18 points higher for participants with some college education, more than 15 points higher for those who graduated HS and almost 9 points higher for those who completed 9th-11th grade. A comparable pattern was observed with respect to DSST scores and participants' family income. These findings show that there is a sizeable SES effect on cognition that may be mediated and they are consistent with several other community-based and population-based studies that have found a strong link between SES, cognitive performance and the risk of dementia and cognitive decline in late life. ${ }^{16}$

The effect of SES on DSST performance was partially mediated by the AL Index, which explained between $1.2 \%$ and $2.9 \%$ of the total effect on cognitive performance for those with HS or greater education and between $2.7 \%$ and $4.5 \%$ for those in the top three PIR quartiles. Although, the null hypothesis concerning the indirect effect was rejected for college educated participants and for those in the top two PIR quartiles, in absolute terms the amount of mediation was relatively small. After controlling for the postulated AL mediator, the DSST Score for college graduates was attenuated by less than 1 point from 24.4 to 23.7, whereas, the DSST Score for those in the highest PIR quartile was reduced from 17.3 to 16.5. For all other SES levels, the reduction was even smaller still at less than half a point. Alternative scoring algorithms for summarising AL burden that took into account participants' prescribed medication use (for diabetes, hypertension and hypercholesterolaemia) and clinical cut-offs as a criterion for 'high risk' did not alter these findings.

Our findings suggest that SES, as measured by educational attainment or family income, is a strong predictor of performance on a test that is sensitive to age and dementiarelated cognitive changes in the elderly. However, only a small proportion of this relationship was mediated by AL. Although, the parameter estimates for the two postulated pathways, through which SES predicts AL and in turn AL predicts performance on the DSST, were statistically significant, their magnitude was modest and their product-reflecting the amount of mediation-was not large enough to account for the substantial impact of SES on cognitive function, which remained largely unaffected after adjusting for AL. Similar findings were obtained when individual components of AL were substituted in place of the summary index. Parameter estimates for paths a and $\mathrm{b}$ associated with each component were generally not of such magnitude as to be considered clinically significant and only WTR and HbAlc were found to have statistically significant mediation effects, although, not of sufficient magnitude to be considered clinically meaningful. Indeed the findings reported here suggest that for a nationally representative sample of US elderly 60 years of age or older, neither cumulative nor individual dysregulations in cardiovascular, metabolic and immune system parameters provide additional information regarding biological pathways through which SES may affect cognitive function. These findings suggest that despite evidence of significant SES-related differentials in biological dysregulation and the likely cumulative nature of these dysregulations across the life course, further efforts are required to elucidate the exact physiological pathways and mechanisms through which SES impacts cognitive function in late life.

Our study used a large, nationally representative sample of elderly, who were assessed through in-person interviews 
and physical examinations, using validated instruments and standardised protocols that remained consistent throughout the 1999-2002 NHANES period. Missing data were multiply imputed using available predictors and a methodology that is well suited to the complex survey design of the NHANES. Moreover, the analyses used a comprehensive index of cumulative biological risk, simultaneous measurements of SES and cognitive function, and controlled for a number of important demographic confounders.

The current study has some important limitations. Because this was a cross-sectional study, we cannot rule out reverse causation, whereby lower cognitive ability may have preceded and was the cause of both lower SES and higher AL scores. Moreover, it is possible that substantive changes in the causal relations in the current mediation model over time could have led to an underestimation of the indirect effect. ${ }^{45}$ For example, studies have found that abnormal values for some of the metabolic and cardiovascular biomarkers used in our AL Index are detrimental in midlife, but not in late life. ${ }^{17}$ A related potential concern for any cross-sectional study on the elderly is that of survivor bias. That is, participants who were eligible and were ultimately enrolled in NHANES may consist of a generally healthier and more resilient cohort of elderly. Therefore, the absence of a stronger link between SES and AL and of AL and cognitive function may reflect such a bias. ${ }^{46}$

We also cannot rule out potential confounding by genes, such as APOE, or psychosocial factors, such as depression, that influence both AL biomarkers and late-life cognitive outcomes. ${ }^{17} 47$ However, confounding by such factors is unlikely given the strength of the SES and DSST relationship. Moreover, the 1999-2002 NHANES did not measure endocrine activity markers, which are hypothesised to play a role in the assessment of AL. ${ }^{31}$ However, even if additional biomarker data was available, currently there is no gold standard approach to measuring $\mathrm{AL}$ and the comparative analyses of AL measurement approaches which have been conducted to date, have shown only modest predictive ability differences among the various indices. ${ }^{48}$ Our study used a single test for cognitive function. A more detailed battery that included additional tests for language, memory, attention and executive function could have provided greater statistical power to detect any AL mediated SES-differences in cognitive function. Lastly, we could not include non-community-dwelling elderly in our analysis, because by design the NHANES excludes all persons in supervised care or custody in institutional settings. Non-community-dwelling elderly individuals would, presumably be more cognitively and physically impaired. As such, their exclusion from our analysis may have biased our findings towards the null.

Despite these potential limitations, the findings reported here suggest that the potential value of the AL Index in mediating SES differences in cognitive function in the elderly is limited. Although numerous studies have shown that individual parameters of cardiovascular, metabolic and immune system function play a role in latelife cognitive function, ${ }^{21}$ our findings suggest that cumulative profiles of biological risk, based on these parameters, do not mediate SES differences in performance on a test that is sensitive to age and dementia-related changes. Given that SES-related adversity affects human health through a multitude of physiological pathways, a more comprehensive view of biological risk may be important in explaining SES differences in mortality or physical health outcomes. ${ }^{25}$ However, our findings do not support the hypothesis that AL is a significant mediator of SESrelated disparities in late-life cognitive function.

Acknowledgements The authors express their gratitude to the participants and staff of the National Health and Nutrition Examination Survey for their invaluable contributions.

Contributors Study design and analyses were conducted by JA who produced the first draft. VH and CZ revised the manuscript for important intellectual content, made contributions to the interpretation of data and approved the final version.

Funding This research was supported by the Alzheimer Disease Research Center at the Icahn School of Medicine at Mount Sinai through a grant from the National Institute on Aging, National Institute of Health (U01 P50 AG005138). VH and $\mathrm{CZ}$ are also supported by the Department of Veterans Affairs, Veterans Health Administration.

\section{Competing interests None declared.}

Patient and public involvement Patients and/or the public were not involved in the design, or conduct, or reporting, or dissemination plans of this research.

\section{Patient consent for publication Not required.}

Ethics approval This study was conducted using National Health and Nutrition Examination Survey (NHANES) data. All NHANES protocols are approved by the National Center for Health Statistics Research Ethics Review Board.

Provenance and peer review Not commissioned; externally peer reviewed.

Data availability statement Data are available in a public, open access repository at https://www.cdc.gov/nchs/nhanes/index.htm

Open access This is an open access article distributed in accordance with the Creative Commons Attribution Non Commercial (CC BY-NC 4.0) license, which permits others to distribute, remix, adapt, build upon this work non-commercially, and license their derivative works on different terms, provided the original work is properly cited, appropriate credit is given, any changes made indicated, and the use is non-commercial. See: http://creativecommons.org/licenses/by-nc/4.0/.

ORCID iD

Jimmy Akrivos http://orcid.org/0000-0002-8872-6652

\section{REFERENCES}

1 Satizabal CL, Beiser AS, Chouraki V, et al. Incidence of dementia over three decades in the Framingham heart study. N Engl J Med 2016;374:523-32.

2 Hebert LE, Weuve J, Scherr PA, et al. Alzheimer disease in the United States (2010-2050) estimated using the 2010 census. Neurology 2013;80:1778-83.

3 Ortman JM, Velkoff VA, Hogan H. An Aging Nation: The Older Population in the United States. Current Population Reports P251140. Washington, DC: U.S. Census Bureau, 2014.

4 Alzheimer's Association Report. 2017 Alzheimer's disease facts and figures. Alzheimers Dement 2017;4:325-73.

5 Weuve J, Hebert LE, Scherr PA, et al. Deaths in the United States among persons with Alzheimer's disease (2010-2050). Alzheimers Dement 2014;10:e40-6.

6 Schneider LS, Mangialasche F, Andreasen N, et al. Clinical trials and late-stage drug development for Alzheimer's disease: an appraisal from 1984 to 2014. J Intern Med 2014;275:251-83.

7 O'Brien JT, Holmes C, Jones M, et al. Clinical practice with antidementia drugs: a revised (third) consensus statement from the British association for psychopharmacology. J Psychopharmacol 2017;31:147-68. 
8 Kane RL, Butler M, Fink HA, et al. Interventions To Prevent AgeRelated Cognitive Decline, Mild Cognitive Impairment, and Clinical Alzheimer's-Type Dementia. Rockville (MD): Agency for Healthcare Research and Quality (US), 2017.

9 Bancks M, Alonso A, Allen N, et al. Temporal trends in cognitive function of older us adults associated with population changes in demographic and cardiovascular profiles. J Epidemiol Community Health 2019;73:612-8.

10 Matthews FE, Arthur A, Barnes LE, et al. A two-decade comparison of prevalence of dementia in individuals aged 65 years and older from three geographical areas of England: results of the cognitive function and ageing study I and II. Lancet 2013;382:1405-12.

11 Schrijvers EMC, Verhaaren BFJ, Koudstaal PJ, et al. Is dementia incidence declining?: trends in dementia incidence since 1990 in the Rotterdam study. Neurology 2012;78:1456-63.

12 Langa KM, Larson EB, Crimmins EM, et al. A comparison of the prevalence of dementia in the United States in 2000 and 2012. JAMA Intern Med 2017;177:51-8.

13 Alley D, Suthers K, Crimmins E. Education and cognitive decline in older Americans: results from the ahead sample. Res Aging 2007;29:73-94.

14 Horvat P, Richards M, Malyutina S, et al. Life course socioeconomic position and mid-late life cognitive function in eastern Europe. $J$ Gerontol B Psychol Sci Soc Sci 2014;69:470-81.

15 Valenzuela MJ. Brain reserve and the prevention of dementia. Curr Opin Psychiatry 2008;21:296-302.

16 Meng X, D'Arcy C. Education and dementia in the context of the cognitive reserve hypothesis: a systematic review with meta-analyses and qualitative analyses. PLoS One 2012;7:e38268.

17 Barnes DE, Yaffe K. The projected effect of risk factor reduction on Alzheimer's disease prevalence. Lancet Neurol 2011;10:819-28.

18 Stern Y. Cognitive reserve in ageing and Alzheimer's disease. Lancet Neurol 2012;11:1006-12.

19 Greenfield EA, Moorman SM. Childhood socioeconomic status and later life cognition: evidence from the Wisconsin longitudinal study. $J$ Aging Health 2019;31:1589-615.

20 Liu Y, Lachman M. Physical and cognitive activity as mediators of the relationship between education and cognition in later life. Innov Aging 2018;2:251.

21 Beeri MS, Ravona-Springer R, Silverman JM, et al. The effects of cardiovascular risk factors on cognitive compromise. Dialogues Clin Neurosci 2009;11:201-12.

22 Hoffman LB, Schmeidler J, Lesser GT, et al. Less Alzheimer disease neuropathology in medicated hypertensive than nonhypertensive persons. Neurology 2009;72:1720-6.

23 Beeri MS, Rapp M, Silverman JM, et al. Coronary artery disease is associated with Alzheimer disease neuropathology in APOE4 carriers. Neurology 2006;66:1399-404.

24 Beeri MS, Schmeidler J, Silverman JM, et al. Insulin in combination with other diabetes medication is associated with less Alzheimer neuropathology. Neurology 2008;71:750-7.

25 Seeman T, Epel E, Gruenewald T, et al. Socio-Economic differentials in peripheral biology: cumulative allostatic load. Ann N Y Acad Sci 2010;1186:223-39.

26 Gruenewald TL, Karlamangla AS, Hu P, et al. History of socioeconomic disadvantage and allostatic load in later life. Soc Sci Med 2012;74:75-83.

27 McEwen BS. Central Role of the Brain in Stress and Adaptation: Allostasis, Biological Embedding, and Cumulative Change. In: Fink G, ed. Stress: concepts, cognition, emotion, and behavior: Handbook in stress series. Cambridge MA: Academic Press, 2016: 1. 39-55.

28 Danese A, Moffitt TE, Harrington $\mathrm{H}$, et al. Adverse childhood experiences and adult risk factors for age-related disease. Arch Pediatr Adolesc Med 2009;163:1135-43.

29 Danese A, McEwen BS. Adverse childhood experiences, allostasis, allostatic load, and age-related disease. Physiol Behav 2012;106:29-39.

30 Lyu J, Burr JA. Socioeconomic status across the life course and cognitive function among older adults: an examination of the latency, pathways, and accumulation hypotheses. J Aging Health 2016;28:40-67.

31 Seeman TE, Singer BH, Rowe JW, et al. Price of adaptation-allostatic load and its health consequences. MacArthur studies of successful aging. Arch Intern Med 1997;157:2259-68.

32 Chyu L, Upchurch DM. Racial and ethnic patterns of allostatic load among adult women in the United States: findings from the National health and nutrition examination survey 1999-2004. J Womens Health 2011;20:575-83.

33 Seeman TE, Crimmins E, Huang $\mathrm{M}-\mathrm{H}$, et al. Cumulative biological risk and socio-economic differences in mortality: MacArthur studies of successful aging. Soc Sci Med 2004;58:1985-97.

34 Hansen Åse M, Andersen LL, Mendes de Leon CF, et al. School education, physical performance in late midlife and allostatic load: a retrospective cohort study. J Epidemiol Community Health 2016;70:748-54.

35 Zipf G, Chiappa M, Porter KS, et al. National health and nutrition examination survey: plan and operations, 1999-2010. National center for health statistics. Vital Health Stat 2013;1.

36 Curtin LR, Mohadjer L, Dohrmann S, et al. The National health and nutrition examination survey: sample design, 1999-2006. National center for health statistics. Vital Health Stat 2012;2.

37 Centers for Disease Control and Prevention. National health and nutrition examination survey, 2019. Available: https://www.cdc.gov/ nchs/nhanes/index.htm [Accessed 2019 May 30].

38 Lezak MD, Howieson DB, Loring DW. Neuropsychological assessment. New York: NY: Oxford University Press, 2004: 368-70.

39 Jaeger J. Digit symbol substitution test: the case for sensitivity over specificity in neuropsychological testing. $J$ Clin Psychopharmacol 2018;38:513-9.

40 Weintraub S, Salmon D, Mercaldo N, et al. The Alzheimer's disease centers' uniform data set (UDS): the neuropsychologic test battery. Alzheimer Dis Assoc Disord 2009;23:91-101.

41 van Buuren S. Multiple imputation of discrete and continuous data by fully conditional specification. Stat Methods Med Res 2007:16:219-42.

42 Baron RM, Kenny DA. The moderator-mediator variable distinction in social psychological research: conceptual, strategic, and statistical considerations. J Pers Soc Psychol 1986;51:1173-82.

43 Sobel ME. Asymptotic confidence intervals for indirect effects in structural equation models. Sociol Methodol 1982;13:290-312.

44 Geronimus AT, Hicken M, Keene D, et al. "Weathering" and age patterns of allostatic load scores among blacks and whites in the United States. Am J Public Health 2006;96:826-33.

45 Cole DA, Maxwell SE. Testing mediational models with longitudinal data: questions and tips in the use of structural equation modeling. $J$ Abnorm Psychol 2003;112:558-77.

46 Crimmins EM, Kim JK, Seeman TE. Poverty and biological risk: the earlier "aging" of the poor. J Gerontol A Biol Sci Med Sci 2009;64:286-92.

47 Mahley RW. Apolipoprotein E: from cardiovascular disease to neurodegenerative disorders. J Mol Med 2016;94:739-46.

48 Seplaki CL, Goldman N, Glei D, et al. A comparative analysis of measurement approaches for physiological dysregulation in an older population. Exp Gerontol 2005:40:438-49.

49 James PA, Oparil S, Carter BL, et al. Evidence-Based guideline for the management of high blood pressure in adults: report from the panel members appointed to the eighth joint National Committee (JNC 8). JAMA 2014;2014:507-20.

50 Expert Panel on Detection, Evaluation, and Treatment of High Blood Cholesterol in Adults. Executive summary of the third report of the National cholesterol education program (NCEP) expert panel on detection, evaluation, and treatment of high blood cholesterol in adults (adult treatment panel III). JAMA 2001;285:2486-97.

51 Millán J, Pintó X, Muñoz A, et al. Lipoprotein ratios: physiological significance and clinical usefulness in cardiovascular prevention. Vasc Health Risk Manag 2009;5:757-65.

52 Jensen MD, Ryan DH, Apovian CM, et al. AHA/ACC/TOS guideline for the management of overweight and obesity in adults: a report of the American College of Cardiology/American heart association Task force on practice guidelines and the obesity Society. Circulation 2013;2013.

53 WHO. Waist circumference and waist-hip ratio. Report of a who expert consultation. Geneva: World Health Organization (WHO), 2008.

54 American Diabetes Association. Glycemic targets. Sec. 6. in standards of medical care in Diabetes-2017. Diabetes Care2017; 40:S48-56.

55 Ridker PM, Page CP. Cardiology patient page. C-reactive protein: a simple test to help predict risk of heart attack and stroke. Circulation 2003;108:e81-5. 